\title{
Acoustic-Emission-Analysis of Dissimilar Laser-welds of Aluminium and Polyamide 6.6
}

\author{
Marc Schiry ${ }^{1}$, Christian Lamberti ${ }^{2}$, Peter Böhm ${ }^{1, *}$, Peter Plapper $^{2}$ \\ ${ }^{1}$ Department of Technology, University of Applied Science Trier, Germany \\ ${ }^{2}$ Faculty of Science, Technology and Communication, University of Luxembourg, Luxembourg
}

Copyright $\mathrm{O} 2016$ by authors, all rights reserved. Authors agree that this article remains permanently open access under the terms of the Creative Commons Attribution License 4.0 International License

\begin{abstract}
A novel laser joining process for hybrid polymer-metal structures has proven a strong bond between polyamide and aluminium. This welding technique is strongly requested by the automobile producing industry for numerous applications within structure components. However, the joining quality exhibits a strong dependency on process-related variables, which highlights the need for an online inspection technique. The requirements for a chosen inspection technique are [1], [2]: 1 . Showing the results on-line during the joining process; 2. Working non-destructive; 3. Working as an integral method; Being able to determine weld defects. In that case only the Acoustic Emission Analysis (AEA) as a Non-Destructive-Technique (NDT) can be used to guarantee reasonable results. During the first tests at the welding laboratory at the Laser Technology Competence Centre (LTCC) at the University of Luxembourg, AEA sensors are applied onto the surface of the test specimen which has to be welded. In this principle acoustic events, caused by welding defects, are recorded during the laser joining. The most important laser process parameters, like velocity, power and horizontal focal position of the laser spot, have been verified and evaluated by the AEA. It was observed that insufficient laser power can lead to the break of the melting bath, which is often not recognizable by non-destructive optical methods [2]. The AEA however was able to detect this lack of fusion. Using high laser power, the appearance of gas bubbles arising from the Polyamide 6.6 could be detected afterwards in a microscopic micrograph. The AEA signals related to this effect can be correlated clearly. Due to the high pressure of the gas bubbles, the aluminium weld is often interrupted, which can be traced back to the solidification of the aluminium molten mass. Due to the actual situation, the AEA is a well-working NDT online monitoring method and can be used for the correlation of acoustic events and welding defects within the mentioned joining method. Future tasks will develop some algorithms to separate the different defects by pattern recognition of the AEA signals and parameter
\end{abstract}

Keywords Acoustic-Sound-Emission, Laser Beam Welding, Aluminium, Polyamide 6.6, Non-destructive Testing

\section{Introduction}

The application of hybrid components offers a high potential in today's manufacturing industry. Particularly the automotive section benefits from the combination of material specific properties such as low weight, high corrosion resistance and thermal conductivity [3]. By joining aluminium to polyamide 6.6 (PA 6.6), the advantages of one material compensate the disadvantages of the other one. For resource efficient manufacturing of lightweight design, the demand of using polymer-metal-products is growing more and more.

In the past, aluminium and PA 6.6 was joined by gluing or thermal joining methods for creating a bonded joint between metal and plastic. Instead of the application of glue, thermal joining induces thermal energy in the joining zone of both materials.

A newly developed laser process for thermal joining of aluminium and PA 6.6 was reported [1]. It works without any filler material. Only a fibre laser with a power of $400 \mathrm{~W}$ is used for producing dissimilar welds. In that case the laser beam is focused several micrometres under the surface of the aluminium specimen [1], [4]. The induced energy heats up the metal to its melting-point of about $660^{\circ} \mathrm{C}$. As a result, the aluminium melting pool transfers the thermal energy by thermal conduction to the PA 6.6. This effect of emerging melted polyamide causes the bonding at the interface of the two materials. Figure 1 shows the schematic sequence of the thermal joining process.

In order to generate a strong and robust physiochemical joint, the surface of the aluminium requires a defined and clean surface. The interaction of reactive molecules segments of the PA and chemical compounds of aluminium, oxygen and hydrogen at the metallic surface creates an 
adhesive joint.

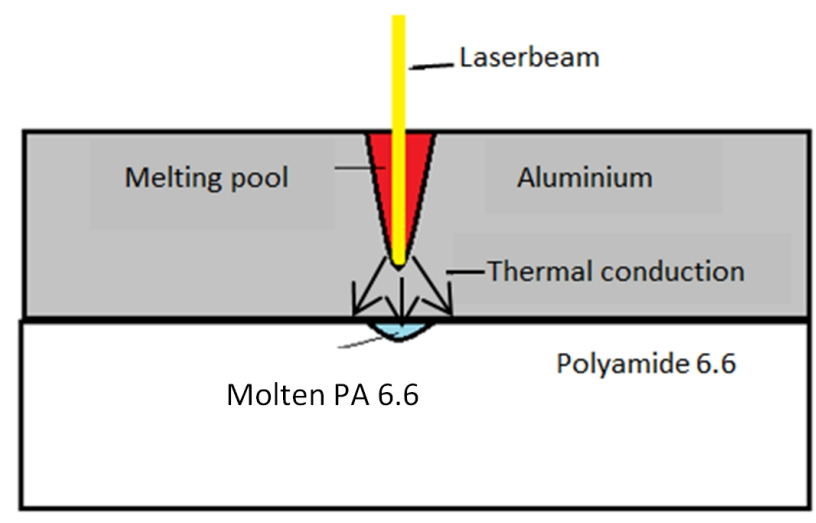

Figure 1. Schematic process

The reaction is taking place in the molten state of the polymer at temperatures above $255^{\circ} \mathrm{C}$ and its thermal decomposition starting below $400^{\circ} \mathrm{C}$. Within this temperature window, the molten polymer is able to connect to the aluminium without a loss of contact area due to the formation of gas bubbles at the interface.

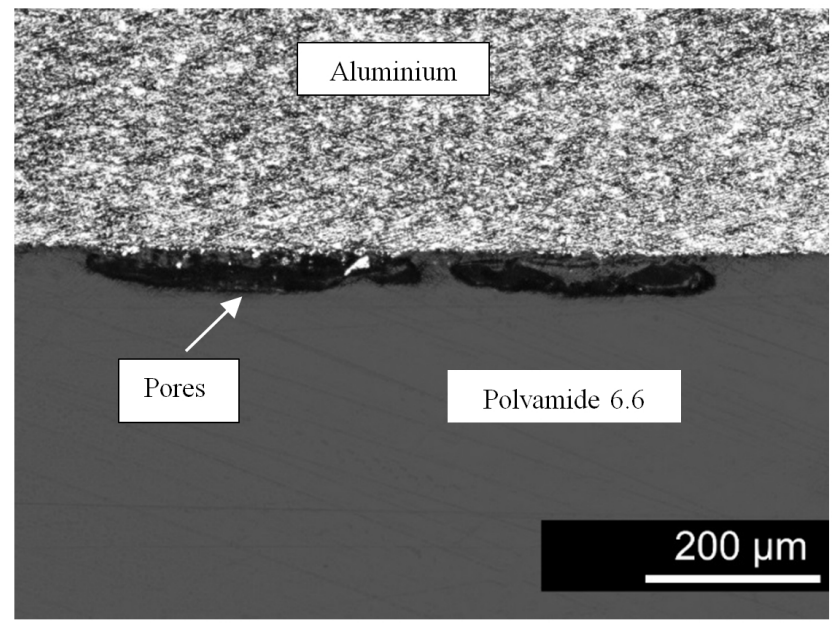

Figure 2. Porosity and connection failures in the interface of aluminum and polyamide

A high number of machine parameters such as the laser power, focal position and timely (pulsed laser) as well as spatial modulation all have a strong impact on the temperature at the interface. All this highlights the requirement to control the process stability. For a qualitative analysis of the thermal joint, destructive analyse techniques are used to obtain information about the strength of the bonded structure. Pulling tests destroy the specimen and micrograph sections are used to study the microstructure of the metal and the plastic joint. However such destructive analysing techniques are not applicable in a real manufacturing situation. In order to guarantee today high quality standards requested by the automotive industry, an overall integrated Non-Destructive-Technique (NDT) is essential in the thermal joining of these distinct dissimilar materials. The Acoustic-Emission-Analysis (AEA) is able to satisfy this demand for a quick, cheap and reliable analysis method. With the investigation of the sound characteristics it is possible to give information about the quality of the welded product [5], [6], [7]. Plastic decomposing, connection failures and porosity (figure 2) can be predicted without an expensive and destructive working analysing method. The AEA presents the economic potential as an online-monitoring solution to be implemented in the manufacturing of dissimilar aluminium and polyamide structures joint by means of laser.

\section{Experimental Setup}

For the experiments, $0.5 \mathrm{~mm}$ thick aluminium sheet material of the type EN-AW1050A was joined to $1 \mathrm{~mm}$ thick polyamide 6.6 using laser power of $380 \mathrm{~W}$ as shown in figure 1.

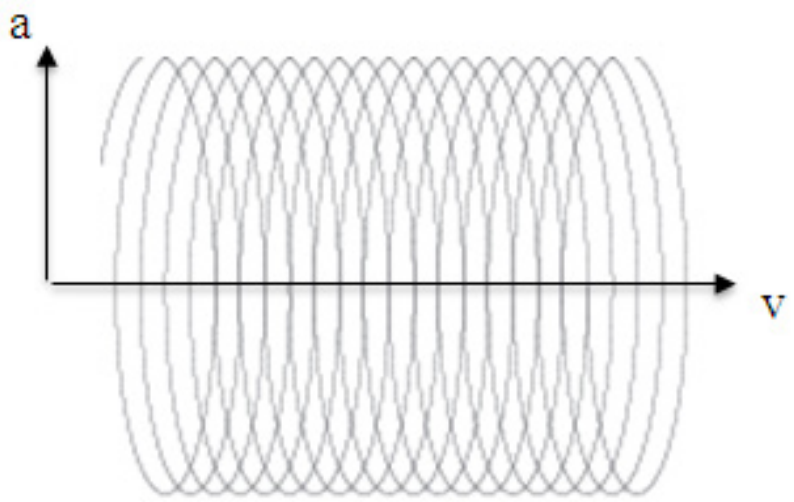

Figure 3. Spatial power modulation by superposed beam oscillation on the linear feed [7]

The joining area was broadened by laser beam oscillation by an amplitude of $0.4 \mathrm{~mm}$ and an oscillation frequency of $500 \mathrm{~Hz}$, figure 2. To limit the penetration depth of the laser beam inside the aluminium, the laser power was further modulated with $25 \mathrm{kHz}$ and a modulation time of $34 \mu \mathrm{s}$.

The vertical focal position is with regard to the limited production accuracy of the single parts and resulted deviated dimensions the most important parameter in the process [1]. In the background of this issue, the focal position was manipulated during this investigation to detect some acoustic signals. Argon gas was used as shielding gas to protect the molten aluminium from oxidation. Activating the inert gas during the welding process is another parameter, which was manipulated in the present work.

The piezo-electric sound sensors were applied onto both sides of the surface of the specimen. Two sensors were fixed by low clamping force and coupled with ultrasonic gel.

The test setup allowed the recording of the signals from both sites for later comparison simultaneously. A suitable trigger threshold was set to filter disruptive noise from the environmental background. 
The signals were characterized by using the Hits-Time-Diagram. This analysing instrument was used to detect defects in the welding process. Single sound occurrences are shown in dependency of their acoustic pressure over the time. The AEA-signals are illustrated in a cloud of green points. Inhomogeneity of the signal could give some evidences for possible defects in the welded zone.

At first it was necessary to produce accurate specimen free from defects for matching the single measure results. The welded parts were observed by using a light optical microscope. Defects in further specimen should be give results to the reference specimen.

\section{Results and Interpretation}
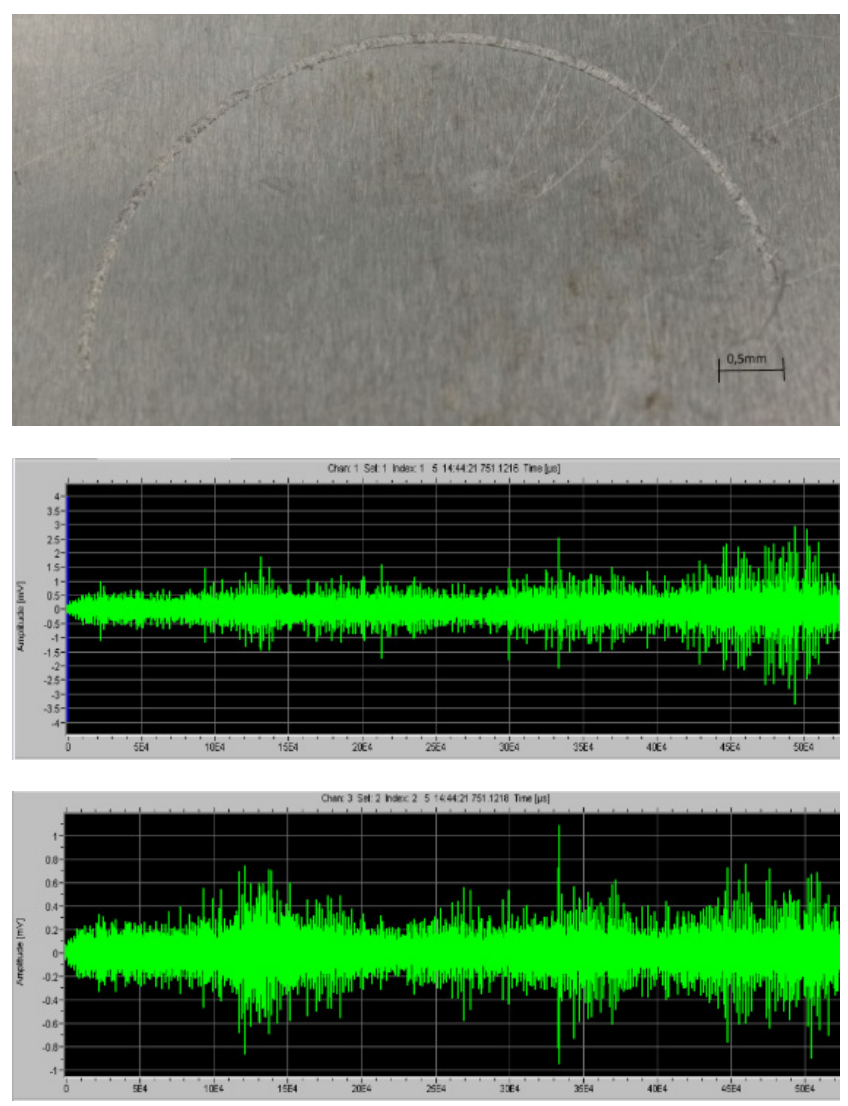

Figure 4. Signals of two different sensors (first aluminium, second PA 6.6)

Theoretically, there is an influence on the measurements in dependency on which side (aluminium or PA 6.6) the senor is fixed. The first measurement considered this issue and was focused on defining the absorption behaviour of the two different materials. For this, the acoustic sensors were applied on both sides on the specimen. The acquired signals of both sensors were compared. Figure 4 shows the difference of these two signals. The signal intensity is noted on the $y$-axis and the time is noted on the $x$-axis. In the first diagram (aluminium sensor) in figure 4 the signal reached in the maximum $2,5 \mathrm{mV}$. The signal of the plastic sensor in figure 4 reached at its maximum only $1 \mathrm{mV}$. This difference in the acoustic signal intensity was caused be the different molecular structure of both materials. Molecular friction between polymer chains dissipates acoustic energy and transforms it into heat. Therefore, the acoustic pressure as well the signal characteristic of the frequency spectrum was curbed. Higher frequencies were damped. This damping behaviour of polyamide is caused by this molecular friction. It collates with the lower sound-speed of polyamide in comparison to aluminium. Due to this effect, the aluminium senor generates a 50\% higher signal than the PA sensor. As a result the piezo-electric sensor was placed on the aluminium sheet during further measurements.

Additional to the influence of the microstructure, the distance of the sensors to the sound source plays a significant rule. With rising distance to the sensor, the signal deceases caused by the damping effect of the microstructure of the two materials. In focus of that issue, it was important to compensate this influence and to perform a constant distance. Therefore, the laser beam was maintained in a circular motion around the sensor by an angle of $180^{\circ}$ (figure 3 ). The task of first investigations was to create a perfect weld, which could be used as reference. In sample 1 the joint shows no defects like pores or instable chemical bond (figure 5).

The recorded signals show a narrow belt of single signals in the Hits-Time-Diagram. In this diagram type, the acoustic pressure is noted on the y-axis and the time is noted on the $\mathrm{x}$-axis. The distribution of the signals was homogeny and consistent. The hits have an acoustic pressure average of $58 \mathrm{~dB}$ (figure 6 ). They are distributed over $12 \mathrm{~dB}$. This sample will be used as a reference for further specimens.

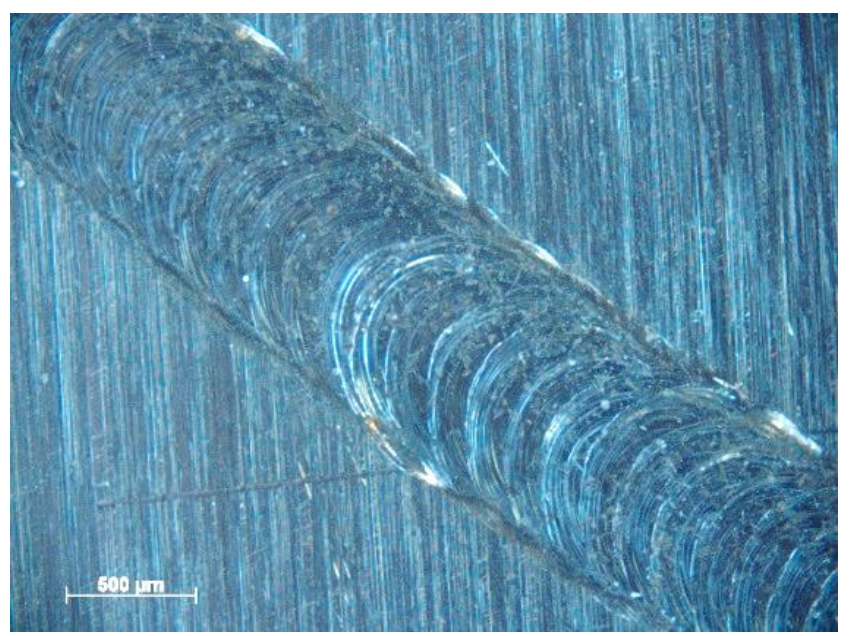

Figure 5. Surface of a good joint in sample 1

If the inert gas was deactivated (no protection of the liquid metal due to oxidation), the melting pool got more instable (figure 7). The result of the higher stream motion of the melt pool affected a rougher surface of the weld seam (figure 7).

The joint of sample 2 was welded with the same vertical focal position as the reference sample. Distinct steam motion of the melting bath and weld spatter results some significant higher AE- signals (figure 8). The average of the acoustic pressure was increased about $10 \mathrm{~dB}$ in the area of the weld 
seam defects, (red rectangle in Figure 8).
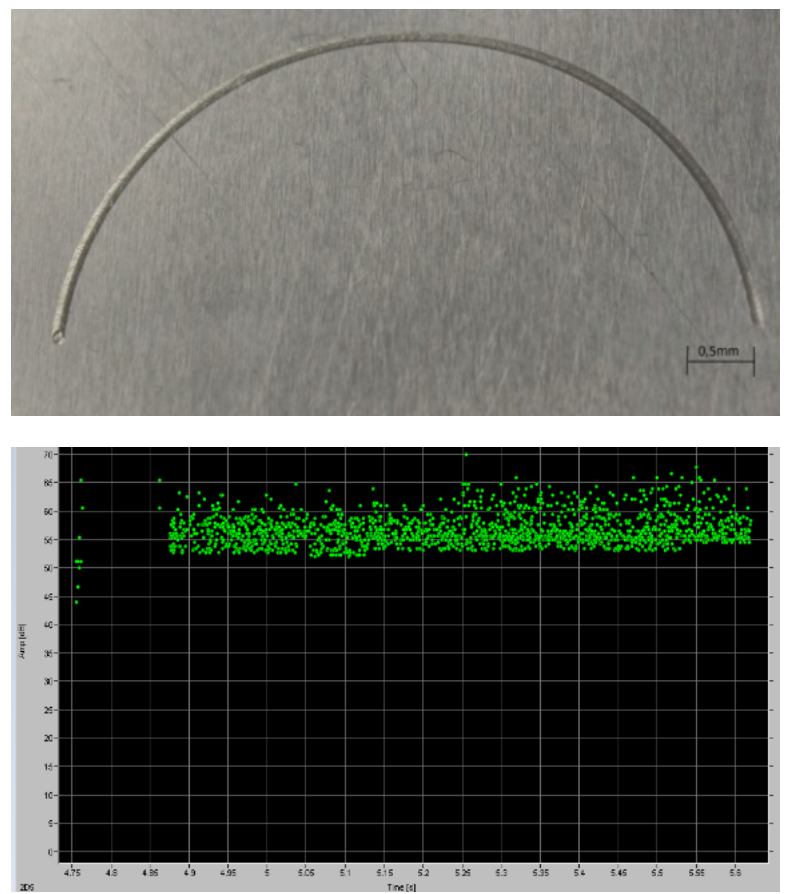

Figure 6. Hits-Time-Diagram sample 1

Sample 2 was welded without any inert gas. The missing gas had no influence on the narrow characteristic of the hit-belt. During welding of specimen 3 the inert gas was also deactivated and the vertical focal position was moved into the aluminium surface. In comparison to the reference sample 1, the laser beam was focused on a higher level in sample 3. Due to that difference, the spot diameter on the surface decreased and the heat density increased. More heat got assumable induced. Figure 9 indicates this causality between the heat input and the formation of bubbles. As a result of the formation of gas bubbles, some gas channels occurred. Their extension reached actually the surface of the plastic sample.

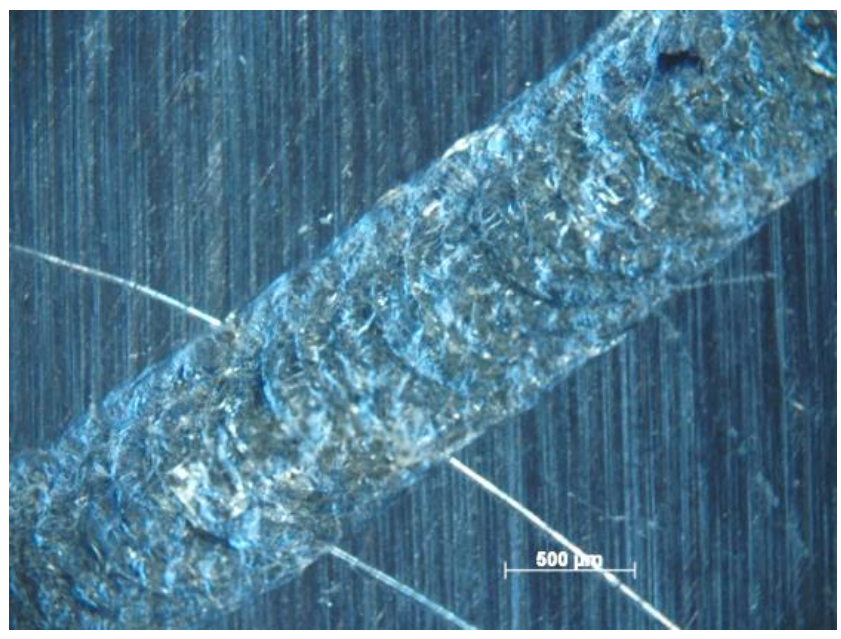

Figure 7. Rough surface of sample 2

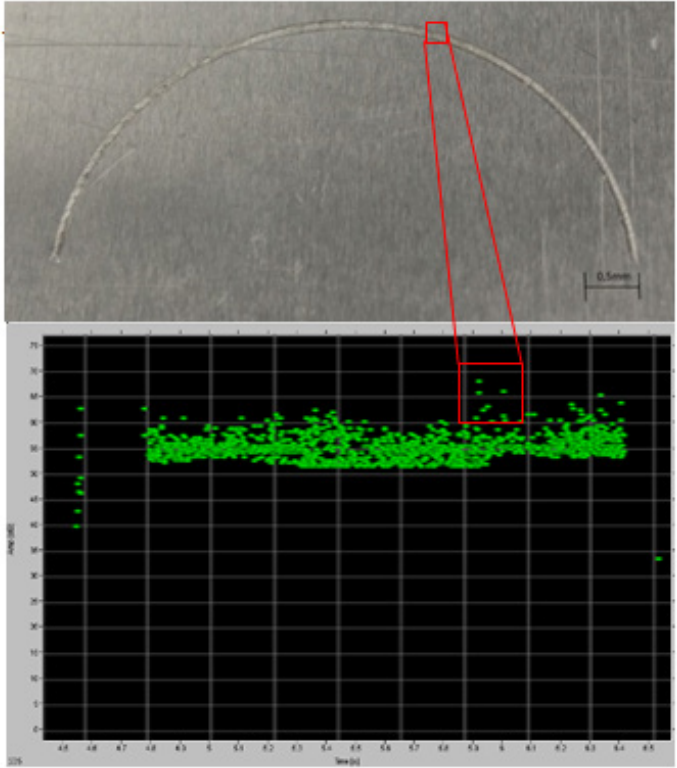

Figure 8. Hits-Time-Diagram sample 2

Analysing the acoustic signals the higher hits in the area of the gas channels are significant and can be correlated with the appearance of the holes. The average acoustic pressure increased in this part of the signal, in which the gas channels appeared, about $10 \mathrm{~dB}$ (Figure 9b).

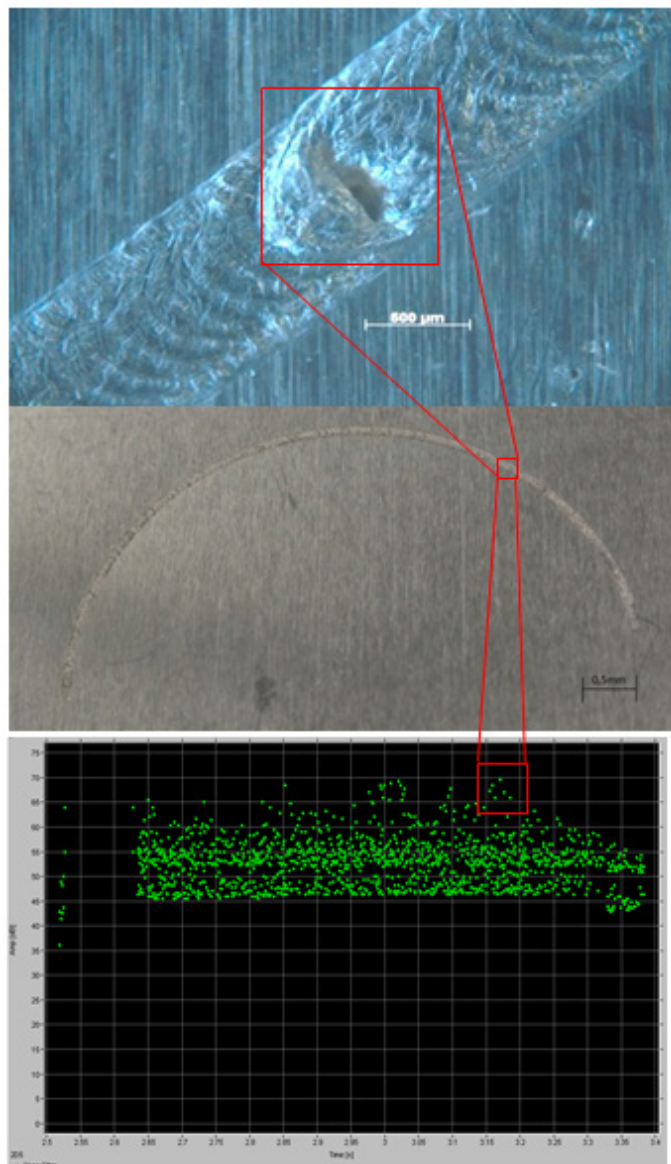

Figure 9. a. Gas channel 9b. Hits-Time-Diagram sample 3 
Further investigations were dealing again with reactivated inert gas, in contrast the vertical focal position of the laser beam has been doubled on the contrary to the reference specimen in sample 4. The acoustic signals reached also double height of the amplitude as a result of the changed focus (figure 10). As a fact of the deeper focal position, the spot diameter of the beam increased on the surface and the heat input was lower than before. The chemical bonding was worse and the pulling strength lower in contrast to the investigations made before.

Sample 5 was focused on the surface of the aluminium. The other parameters have been fixed. Due to the minimized laser beam spot diameter, the thermal heat input reached its maximum. The plastic started its decomposition and as a result micro bubbles, nicks, gas channels and weld spatter occurred (Figure 11b).

The Hits-Time-Diagram shows an irregular signal cloud. Some of the signals were scattered widely. The result was a weak joint with low pulling strength.
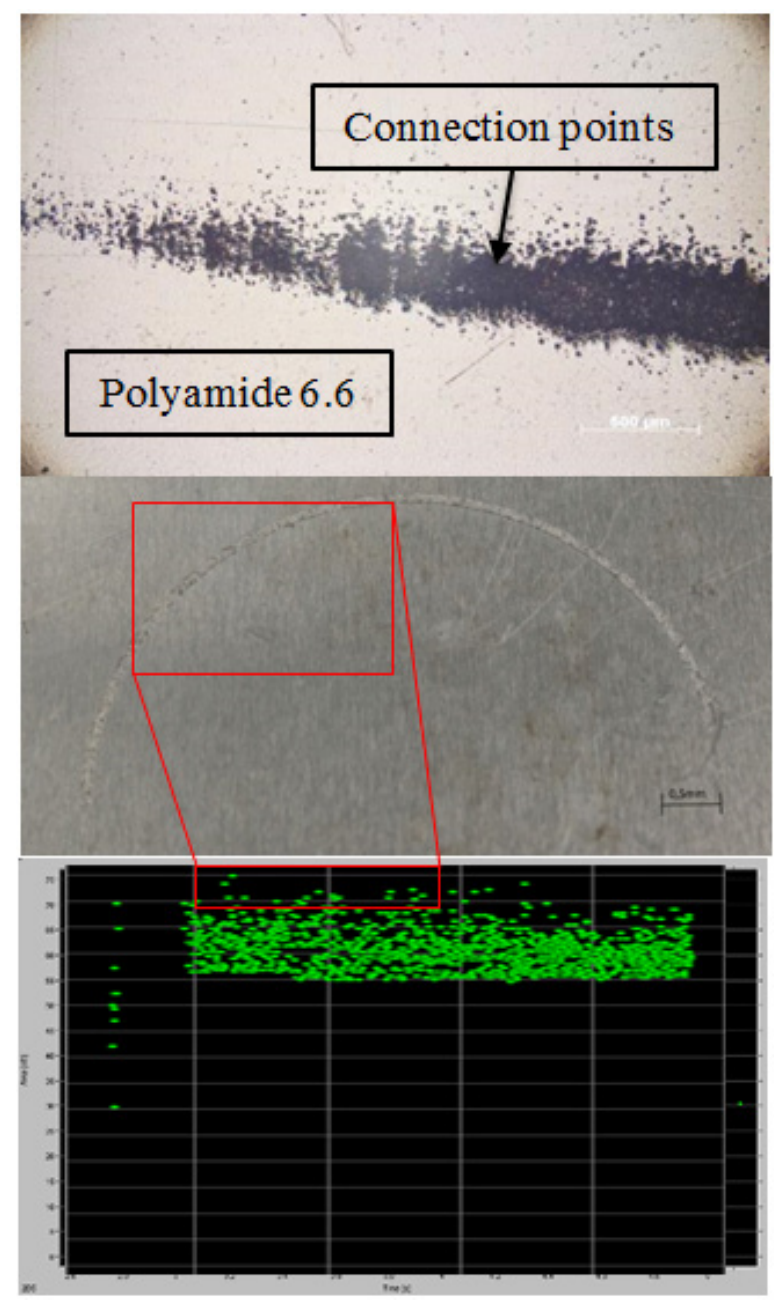

Figure 10. a. Surface PA 6.6 , b. Hits-Time-Diagram sample 4

A comparison of sample 5 to sample 4 shows the dependency of the signal intensity and acoustic pressure to the vertical focal position of the laser spot. A deeper focus beam affects an increasing acoustic pressure. The reason for this issue is caused by the bigger melting pool depending on the deeper focal position.
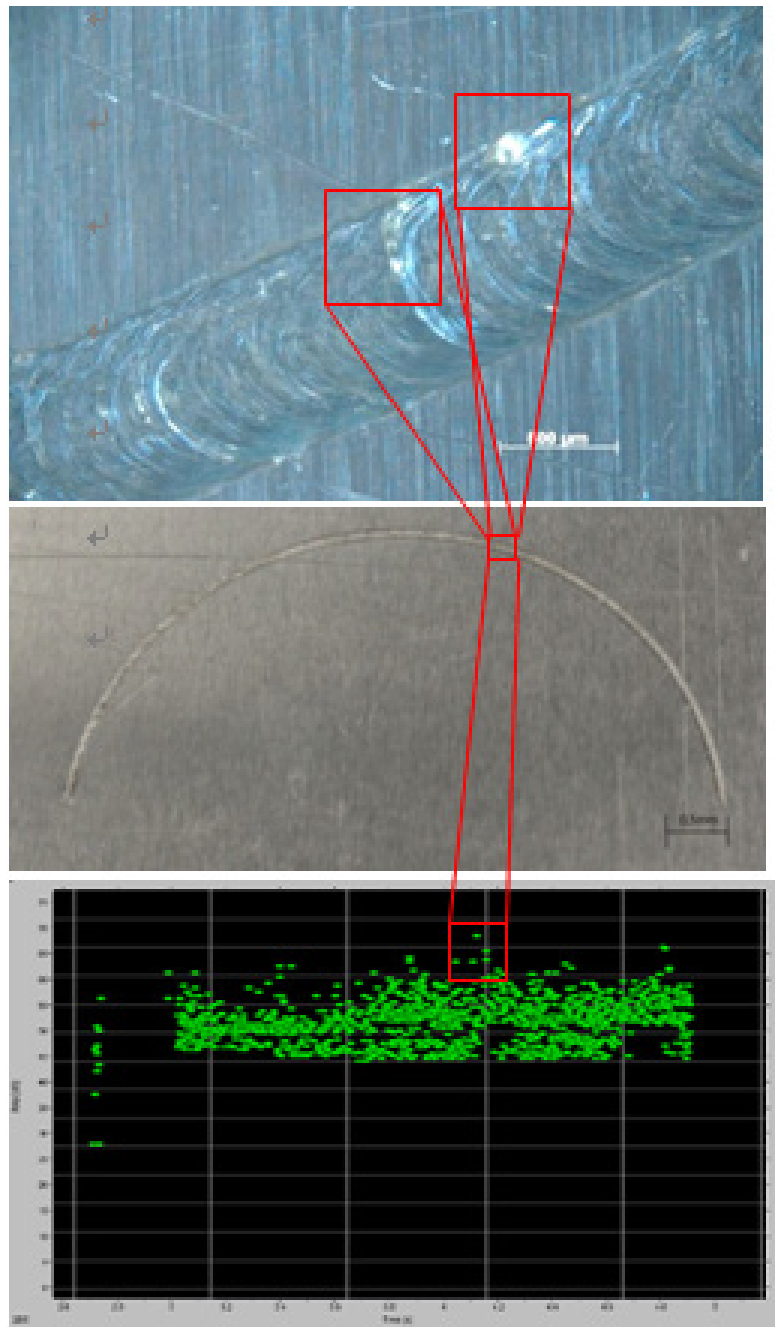

Figure 11. a. Surface PA 6.6 ; b. Hits-Time-Diagram sample 5

\section{Summary}

After the present test campaign, the Acoustic Emission Analysis has proven to be a useful instrument for detecting weld seam defects of metal polymer joints. Using this method, it is possible to identify most of the potential defects, like pores, gas channels and insufficient chemical bonds, without the requirement of destroying the samples to check their ability by destructive tests.

During this work, it was shown that the material of the joining partner has a big influence on the measurement results. Concerning the depressant characteristic of the polymer, an application on the PA 6.6 is not useful. In addition to that, the distance of the sensor to the weld spot has a strong influence to the signal. To compensate this parameter, the laser beam was maintained in a circular motion with a constant distance to the transducer. Further investigations have to deal in more detailed way with regard to the influence of the distance and the thickness of the 
specimens.

The dependency of the signal intensity to the vertical focal position was shown in this study. Increasing depth of the focus affects a higher acoustic pressure followed by higher signal intensity. Therefore the size and the depth of the keyhole is main reason for this causality.

Single defects like gas channels, pores, nicks and weld spatter could be detected by the Hits-Time-Diagram. Defects caused by low connection rate between the aluminium and the PA 6.6 were detected not in any case. The additional process noise created signals which adulterated the diagram and made a meaningful result quiet difficult. For further investigations it would be important to develop a filter algorithm in view of a cluster analysis to isolate the defect signals from the process signals.

However, the Acoustic-Emission-Analysis is a beneficial instrument for Non-Destructive-Testing of dissimilar joints, like plastic and metall. With this technology, it is possible to detect process instabilities during the welding procedure. Post process investigations with destructive techniques are no longer required. After further improvement, Acoustic-Emission-Analysis could be used as monitoring tool in manufacturing of laser welded plastic-metal joints.

\section{REFERENCES}

[1] C. Lamberti, „Laserbeam welding of dissimilar materials in case of polyamide 6.6 and aluminium“, Masterthesis University of Applied Science Trier, 2013

[2] Böhm, Peter, Plapper, Peter, Lamberti, Christian, Neumann, Leonhard (2015), „On-line monitoring of the laser joining process of Aluminium to a thermoplastic resin", Yearly report of the University of Applied Sciences Trier, Teaching and Research 2015

[3] Jung, Kwang-Woon, et al. (2013). „Laser direct joining of carbon fibre reinforced plastic to aluminium alloy", Journal of Laser Applifications 25.3: 032003

[4] T. Solchenbach, , Laser based braze-welding of dissimilar copper-aluminium joints“, Dissertation, Luxembourg, 2014

[5] Ismail, Zubaidah, et al. (2013) „Acoustic emission signals detected during the resistance spot welding of aluminium alloy“, Measurements 46.3 (2013): 1053-1060

[6] H.A. Crostack, H.D. Steffen, H.J. Storp, J. Kolleck, P. Böhm, G. Schulze, P. Jax und V. Streicher, „Acoustic-Emission-Analysis “, DGZfP, Berlin, 1990

[7] P. Böhm, „Acoustic Emission Inspections of Nuclear Components Considering Recent Research Programmes", Charlotte, USA20/23.03, 1989, Symposium: World Meeting on Acoustic Emission, Acoustic Emission-current practice and future directions, Sachse/ Roget/ Yamaguchi ASTM SPT 1077, ASTM pub. Code (PCN) 04-010770-22, Baltimore, S. 78/83, 1991

[8] Amend, P., Pfindel,S., Schmidt, M. (2013) „Thermal joining of thermoplastic metal hybrids by means of mono- and polychromatic radiation", Technical Report, Bayrisches Landeszentrum $\mathrm{GmbH}$. 